\title{
Low Intensity laser therapy in patients with burning mouth syndrome: a randomized, placebo-controlled study
}

\section{Norberto Nobuo SUGAYA(a) Érica Fernanda Patrício da SILVA ${ }^{(a)}$ Ilka Tiemi KATO ${ }^{(b)}$ Renato PRATES(b) Camila de Barros GALLO(a) Vivian Diane PELLEGRINI(a)}

(a) Universidade de São Paulo - USP, School of Dentistry, Department of Stomatology, São Paulo, SP, Brazil.

(b) Instituto de Pesquisa Nuclear - IPEN, São Paulo, SP, Brazil.

Declaration of Interests: The authors certify that they have no commercial or associative interest that represents a conflict of interest in connection with the manuscript.

Corresponding Author: Norberto Nobuo Sugaya E-mail:nnsugaya@usp.br

DOI: 10.1590/1807-3107BOR-2016.vol30.0108

Submitted: Nov 18, 2015

Accepted for publication: Jun 07, 2016

Last revision: July 24, 2016

\begin{abstract}
The aim of this study was to assess the effectiveness of low intensity laser therapy in patients with Burning Mouth Syndrome (BMS). Thirty BMS subjects were randomized into two groups - Laser (LG) and Placebo (CG). Seven patients dropped out, leaving 13 patients in LG and 10 patients in CG. Each patient received 4 irradiations (laser or placebo) twice a week, for two consecutive weeks (blinded to the type of irradiation received). Infrared laser (AsGaAI) irradiations were applied to the affected mucosa in scanning mode, wavelength of $790 \mathrm{~nm}$, output power of $20 \mathrm{~mW}$ and fluence of $6 \mathrm{~J} / \mathrm{cm}^{2}$. A visual analogue scale (VAS) was used to assess the therapeutic effect before and after each irradiation, and at all the control time periods: 7, 14, 30, 60 and 90 days after the last irradiation. One researcher delivered irradiation and another recorded the results. Both researchers were blinded, the first to the results, and the second to the type of radiation applied. The results were categorized according to the percentage of symptom level variation, and showed a statistically better response in LG in only two categories of the control checkpoints ( $p=0.02$; Fisher's Exact Test). According to the protocol used in this study, low intensity laser therapy is as beneficial to patients with BMS as placebo treatment, indicating a great emotional component of involvement in BMS symptomatology. Nevertheless, there were positive results in some statistical analyses, thus encouraging further research in BMS laser therapy with other irradiation parameters.
\end{abstract}

Keywords: Burning Mouth Syndrome; Laser Therapy; Glossalgia.

\section{Introduction}

Burning mouth syndrome is a distinct nosological entity characterized by a chronic idiopathic burning sensation that affects the oral cavity mucosa, without presenting any local clinical signs or detectable laboratory alterations ${ }^{1}$. It usually affects individuals between the $4^{\text {th }}$ and $5^{\text {th }}$ decade of life, mainly women, seven times more often than men $^{2}$. The prevalence is $0.7 \%$ to $4.6 \%$ in the general population ${ }^{3,4}$. Burning mouth symptom is commonly followed by dysgeusia (persistent metallic or bitter taste in the mouth) and xerostomia ${ }^{5,6}$. The most commonly affected sites are the tongue, palate, mucosa of the upper lip and buccal mucosa ${ }^{7}$.

The unknown etiology of BMS has led to the investigation of a series of possible local and systemic causal factors, such as hyposalivation, 
oral candidiasis, anemia, nutritional deficiencies, hyperglycemia and hormonal disturbances associated with menopause ${ }^{7}$. Variations in the psychological profile of these patients have also deserved attention. Anxiety, depression and cancerophobia are quite evident psychological behaviors in patients with BMS8, although no cause-and-effect relationship between psychological disturbance and BMS manifestation has been established ${ }^{9,10}$. A possible etiologic relationship of BMS with the central nervous system or peripheral nervous system has also been studied. Investigations showing alterations in the eyeblink reflex and abnormalities in quantitative sensory testing and electrophysiological tests have suggested a possible neuropathy in peripheral innervation as being the cause of BMS ${ }^{11}$. Jääskeläinen ${ }^{12}$, classifies BMS patients into three subgroups, according to different neurogenic factors. These subgroups are clinically indistinguishable and require neurophysiologic, psychophysical, neuropathological and functional imaging studies to be identified. The first subgroup (50-65\%) would present peripheral, smalldiameter fiber neuropathy of oral mucosa; the second (20-25\%), subclinical trigeminal system pathology; and the third $(20-40 \%)$ would be attributed to a central pain related to the hypofunction of dopaminergic neurons.

Biopsy of tissues affected by the burning sensation showed a reduction in the density of epithelial and sub-papillary nerve fibers, suggestive of axonal degeneration and sensory neuropathy in the lower branches of the trigeminal nerve ${ }^{13}$. Hypothetically, dysfunction of the chorda tympani nerve could cause lingual nerve hyperfunction, and, consequently, a burning sensation ${ }^{14}$. In the field of molecular biology, calcitonin gene-related peptide levels (CGRP) in the saliva of patients with BMS have been found to be lower than those of control groups ${ }^{15}$. Calcitonin gene-related peptide (CGRP) modulates nociceptive trigeminovascular transmission.

Present treatment proposals are still empirical and mostly represented by therapies prescribed to control neuropathic pain ${ }^{8}$. Medications such as tricyclic antidepressants, benzodiazepines, antiepileptic and gabapentin are directed primarily at controlling the symptoms and not at curing the disease ${ }^{1}$. Capsaicin seems to produce favorable results in the treatment of
BMS; however, there are few clinical trials to provide adequate support for this therapeutic agent as a first line of medication to treat BMS patients ${ }^{16}$. Alpha-lipoic acid (ALA) is an important antioxidant that prevents oxidative damage to the nervous system, but, in a randomized, double-blinded, controlled trial in patients with BMS, there was no statistically significant difference between ALA and placebo groups ${ }^{17}$.

A case series of BMS patients (8 patients and 21 affected sites) showed $65 \%$ improvement in symptoms after irradiation sessions with low intensity laser therapy ${ }^{18}$.

The aim of this research was to assess the effect of low intensity laser therapy in patients with burning mouth syndrome, according to a randomized, placebo-controlled study.

\section{Methodology}

\section{Ethical issues}

This study was approved by the Research Ethics Committee of the Sao Paulo University School of Dentistry (FR-224649; 177/08), and developed according to the ethical principles for medical research involving human subjects, stated by the Declaration of Helsinki.

\section{Patients}

Patients affected by burning mouth syndrome were selected at the Stomatology Clinic of the Sao Paulo University School of Dentistry. The participants of this study met the diagnostic criteria for BMS, and agreed to the terms of the informed consent.

\section{Exclusion Criteria}

The following exclusion criteria were adopted in this study: clinical alterations in the oral mucosa potentially associated with the burning symptom, hyposalivation, diabetes, B hypovitaminosis and anemia. Bearing in mind the laser radiation, patients with history of malignant or benign head and neck neoplasia, as well as pregnant and breastfeeding women were not considered eligible for this study.

\section{Randomization}

The patients were divided into two groups: Group 1 - LASER (LG) and Group 2 - CONTROL (CG). 
Allocation between the two groups was randomized by a computer program (20), and the allocation sequence was concealed in serially numbered envelopes (prepared by an independent researcher), which were revealed to the main researcher at the time of irradiation delivery (laser or placebo). All patients, irrespective of the allocation group, fulfilled the same attendance protocol.

\section{Treatment and blinding strategy}

The main researcher was responsible for delivering the irradiation doses. The type of irradiation to be given was determined at the time of delivery, according to the allocation strategy.

Patients were blinded to the nature of irradiation received. Results were assessed by an auxiliary researcher, using a visual analogue scale and a global perception chart. The auxiliary kept the main researcher blind to this information, whereas the researcher responsible for collecting the results was blinded to the type of irradiation delivered to the patients.

\section{Material}

The appliance used was a low intensity infrared diode laser: AsGaAl, model QTUM00A/QUANTUM (Ecco Fibras Opticas e Dispositivos LTDA), according to the specifications shown in Chart 1.

\section{Method}

Participants in this study were submitted to a complete clinical exam, consisting of anamnesis, physical exam, non-stimulated sialometry and hematological tests (complete blood count, fasting glucose, serum ferritin level, vitamin B12 and folic acid investigation), in order to establish a sound diagnosis for BMS and rule out other conditions that could be associated with the burning mouth sensation (diabetes, anemia, hypovitaminosis). The patients were instructed not to use any other type of treatment for burning mouth symptomatology during the study.

The non-stimulated salivary flow was assessed by means of a 15-minute collection time in the morning, about two hours after breakfast. A volume below $0.1 \mathrm{ml} / \mathrm{min}$ was characterized as hyposalivation, thus excluding the individual from the study.

Laser or placebo irradiation was delivered in scanning mode with the laser point in contact with the mucosa. The energy released was $6 \mathrm{~J} / \mathrm{cm}^{2}$, and irradiation was applied on the entire area affected by the burning sensation. The procedures adopted in the placebo group were identical, but no laser energy was delivered. Irradiation time was determined by the extension of the affected area, according to the following equation:

$$
\begin{gathered}
\mathrm{t}=\mathrm{D} \times \mathrm{A} \\
\mathrm{P}
\end{gathered}
$$

Where $\mathrm{t}=$ time $(\mathrm{s}), \mathrm{D}=$ energy density $\left(\mathrm{J} / \mathrm{cm}^{2}\right)$, $\mathrm{A}=$ area and $\mathrm{P}=$ power $(\mathrm{W})$.

The region affected by the burning sensation was delimited with a red dermographic pencil, according to the symptomatology informed by the patient. The demarcated area was transferred to

Chart 1. Characteristics of the laser radiation applied.

\begin{tabular}{lc}
\hline 1. Equipment & Low intensity laser - QTUMOOA/QUANTUM (Ecco Fibras Ópticas e Dispositivos LTDA) \\
$\begin{array}{lc}\text { 2. Wavelength } & 6 \mathrm{~J} / \mathrm{cm}^{2} \\
\text { 3. Fluency } & 120 \mathrm{~mW} \text { or } 0.12 \mathrm{~W} \\
\text { 4. Power } & 0.03 \mathrm{~cm}^{2} \\
\text { 5. Beam area } & 4 \mathrm{~W} / \mathrm{cm}^{2} \\
\text { 6. Power density, radiation or intensity } & 6 \mathrm{~J} \\
\text { 7. Energy per point } & 50 \mathrm{~s} \\
\text { 8. Application time per point } & \text { Variable according to irradiated area } \\
\text { 9. Total number of points } & 1 \mathrm{~cm}^{2} \\
\text { 10. Distance between points } & \text { Variable according to irradiated area } \\
\text { 11. Total time } & \text { Continuous mode } \\
\text { 12. Frequency } & \end{array}$
\end{tabular}


graph tracing paper to allow determination of the area to be irradiated in $\mathrm{cm}^{2}$. Patients underwent four sessions of irradiation, two per week, with a 3-day interval between each session. Placebo irradiation was delivered with the timer beep kept active and the laser source, inactive, so that the patient heard the "beep" at regular intervals without receiving laser irradiation, for the period determined by the same equation used for the laser group. At the end of the study, the patients who showed no improvement were referred to another treatment in progress at our institution.

\section{Recording the results}

A visual analogue scale (VAS) was used to assess the intensity of burning sensation. VAS was applied before and 15 minutes after each irradiation, and at all established control time intervals: 7, 14, 30, 60 and 90 days after the last irradiation.

\section{Statistical analysis}

Statistical analysis was conducted by a qualified statistician blinded to the patient groups. The data sent for analysis were identified only as belonging to Group A or Group B of patients.

\section{Results}

Forty-three patients eligible for this study, who complained of burning mouth, were examined at the Oral Diagnosis Clinic of our institution in the last two years. Thirteen patients were excluded due to the exclusion criteria used in this study (4 due to hypertension, 4 due to candidiasis, 3 due to hypovitaminosis, and 2 due to diabetes). Thirty patients were randomized between the two experimental groups: LG and CG. During the course of treatment, seven patients dropped out: two patients in the laser group and five in the placebo group (Figure 1).

Twenty-three individuals completed the research protocol: two men and twenty-one women, with a mean age of 59.7 years (29-83). Mean duration of symptomatology was 31.7 months (6-192). The triad of burning mouth syndrome symptoms, characterized by xerostomia, dysgeusia and burning sensation, was common among the patients. The sites affected by BMS identified in the sample were as follows: tongue, lower lip, upper lip, buccal mucosa, mandibular ridge, palate and mandibular gingiva, involving 24 LG sites and 17 CG sites (Table 1).

VAS figures were transformed into percentages of symptoms, in order to enable statistical tests (Fisher's Exact Test) without involving negative values.

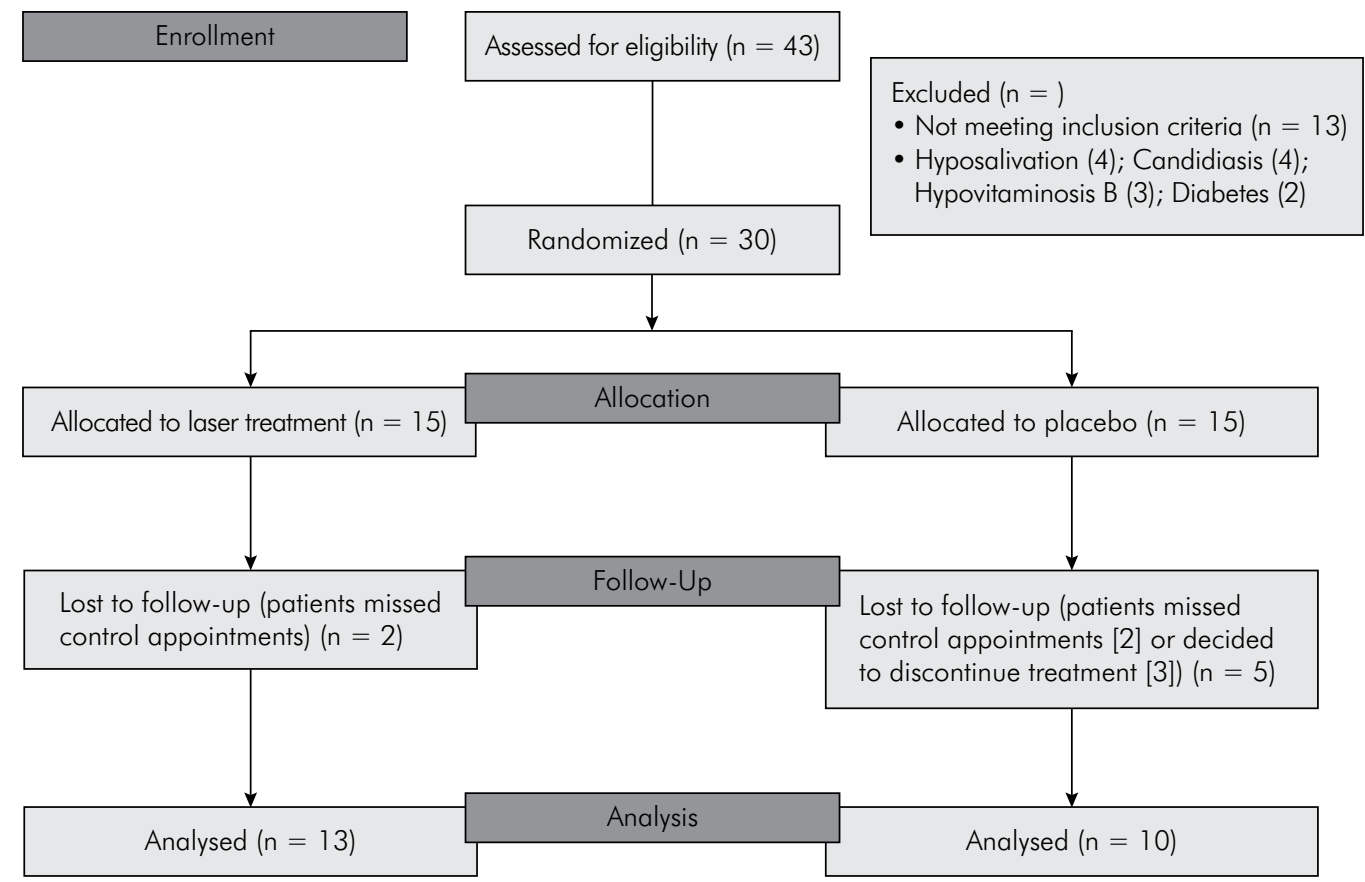

Figure 1. Consort flow diagram 
Therefore, the initial VAS figure was defined as 100\% of symptomatology and the later assessments were calculated accordingly, showing figures towards zero when symptomatology improved, and above 100 when condition worsened during the course of the study. Patients were categorized according to their percentage of symptoms at the end of the last control period, as follows: NB (no burning sensation) - patients who presented $0 \%$ symptomatology; $\mathrm{E}$ (excellent) - patients with $1 \%$ to $25 \%$ symptomatology, G (good) - $26 \%$ to $50 \%$, $\mathrm{R}$ (regular) - $51 \%$ to $75 \%$, U (unchanged) - $76 \%$ to $100 \%$, and $\mathrm{W}$ (worsened) - values above $100 \%$ (Charts 2 and 3 ).

Table 1. Patients' clinical profile.

\begin{tabular}{lccc}
\hline Variable & Laser group & Control group & Total \\
\hline Men & 0 & 2 & 2 \\
Women & 13 & 8 & 21 \\
Mean age (range) & $57.3(29-83)$ & $62.7(53-81)$ & $59.7(29-83)$ \\
Mean duration of BMS symptoms (months) & $25.5(6-192)$ & $39.6(6-180)$ & $31.7(6-192)$ \\
Xerostomia complaint (n) & 6 & 1 & 7 \\
Dysgeusia complaint (n) & 1 & 3 & 4 \\
Dysgeusia and xerostomia complaint & 3 & 5 & 7 \\
Previous treatment for BMS (n) & 9 & 6 & 14 \\
Patients with 1 affected site (n) & 5 & 1 & 11 \\
Patients with 2 affected sites $(n)$ & 5 & 3 & 6 \\
Patients with 3 or more affected sites $(\mathrm{n})$ & 3 & & 6 \\
\hline
\end{tabular}

Chart 2. VAS values after each irradiation session and in the five control checkpoints in Laser Group expressed according to the categories of outcome.

\begin{tabular}{lcccccccccccccc}
\hline GL & P1 & P2 & P3 & P4 & P5 & P6 & P7 & P8 & P9 & P10 & P11 & P12 & P13 \\
I1 & 1 & 5 & 3 & 5 & 3 & 4 & 2 & 5 & 2 & 5 & 4 & 2 & 2 \\
I2 & 0 & 5 & 1 & 2 & 0 & 5 & 3 & 5 & 2 & 5 & 2 & 1 & 0 \\
I3 & 0 & 3 & 0 & 1 & 5 & 5 & 1 & 0 & 1 & 4 & 3 & 1 & 0 \\
I4 & 1 & 3 & 1 & 2 & 3 & 5 & 1 & 0 & 3 & 5 & 3 & 0 & 1 \\
C1 & 0 & 4 & 1 & 1 & 5 & 5 & 1 & 5 & 0 & 5 & 3 & 0 & 1 \\
C2 & 0 & 4 & 0 & 1 & 2 & 5 & 1 & 0 & 0 & 5 & 3 & 1 & 2 \\
C3 & 0 & 5 & 1 & 1 & 5 & 5 & 1 & 0 & 0 & 4 & 3 & 1 & 2 \\
C4 & 0 & 5 & 1 & 1 & 5 & 4 & 1 & 0 & 0 & 4 & 1 & 0 & 4 \\
C5 & 0 & 5 & 0 & 1 & 5 & 5 & 1 & 0 & 0 & 5 & 5 & 0 & 0 \\
\hline
\end{tabular}

P: patient; 11-4: irradiations sessions 1 to 4; C1-5: control checkpoints 1 to 5; 0: no burning sensation (0\%); 1: excellent (1-25\%); 2: good (26-50\%); 3: regular (51-75\%); 4: unchanged (76-100\%); 5: worsened (> 100\%)

Chart 3. VAS values after each irradiation session and in the five control checkpoints in Control Group expressed according to the categories of outcome.

\begin{tabular}{lccccccccccc}
\hline GP & P1 & P2 & P3 & P4 & P5 & P6 & P7 & P8 & P9 & P10 \\
I1 & 1 & 4 & 5 & 4 & 2 & 2 & 4 & 2 & 2 & 2 \\
I2 & 1 & 3 & 5 & 4 & 0 & 1 & 4 & 1 & 3 & 3 \\
I3 & 1 & 2 & 4 & 3 & 0 & 1 & 4 & 3 & 4 & 3 \\
I4 & 0 & 3 & 5 & 0 & 0 & 0 & 4 & 4 & 2 & 3 \\
C1 & 1 & 2 & 5 & 2 & 0 & 1 & 4 & 4 & 2 & 2 \\
C2 & 1 & 2 & 5 & 0 & 0 & 0 & 4 & 3 & 1 & 1 \\
C3 & 1 & 1 & 5 & 0 & 0 & 0 & 4 & 5 & 1 & 1 \\
C4 & 1 & 3 & 5 & 3 & 0 & 0 & 4 & 5 & 0 & 1 \\
C5 & 1 & 3 & 4 & 0 & 0 & 1 & 4 & 5 & 0 & 0 \\
\hline
\end{tabular}

P: patient; 11-4: irradiations sessions 1 to 4; C1-5: control checkpoints 1 to 5; 0: no burning sensation (0\%); 1: excellent (1-25\%); 2: good (26-50\%); 3: regular (51-75\%); 4: unchanged (76-100\%); 5: worsened (>100\%). 
Six of the 13 patients from the laser group reported complete remission of symptoms in all sites affected by the burning sensation at the last control checkpoint, 90 days after the last irradiation. Seven patients presented complete remission of symptoms in some of their affected sites at that same checkpoint. According to the sites affected in the laser group, 15 out of 24 were classified as no burning at the last control checkpoint (Figure 2).

Four of the 10 patients that composed the placebo group reported total remission of symptoms in all affected sites at the end of the control period. Four of the 16 affected sites presented complete remission of symptoms at the 90-day control checkpoint, after the last irradiation (Figure 2).

When comparing the results between the laser and the placebo groups by response categories, the categories that showed statistical significance were E in the last irradiation (0.002) and NB at the control checkpoint of 90 days (0.02), in favor of the laser group.

\section{Discussion}

Burning mouth syndrome (BMS) is thought to be a neuropathic disease ${ }^{12,13}$; nevertheless, its exact causes and proper treatment are still unknown.

Therapy for BMS is still unsatisfactory, and there are few evidence-based findings. On the other hand, there are patients in need of relief from BMS symptoms. The historical progression of this condition has traversed different periods in regard to patient management, according to different etiological theories that have been suggested. This is how it has always been, ever since the existence of the disease was refuted and BMS symptomatology was attributed to psychiatric disorders, up until today, when the main theory points to a possible neurogenic etiology.

The present research did not intend to clarify the basis of BMS etiopathology; nonetheless, the theory of the neuropathic origin of BMS, and the recognized properties of laser therapy were taken into account in designing the treatment protocol tested in this study. There is evidence of peripheral nerve involvement, such as the chorda tympani nerve ${ }^{14}$, and reduction in the density of lingual innervations in BMS patients ${ }^{13}$.

The choice of laser therapy to treat BMS patients was based on the regenerative properties of low intensity laser on nerve fibers ${ }^{19,20,21,22}$. A previous pilot study showed positive results and encouraged the development of this controlled clinical trial ${ }^{18}$.

Chow et al. ${ }^{23}$, showed that parameters of laser irradiation used in the present study reduced acute and chronic pain by changing mitochondrial signaling and reducing ATP availability. According to these authors,

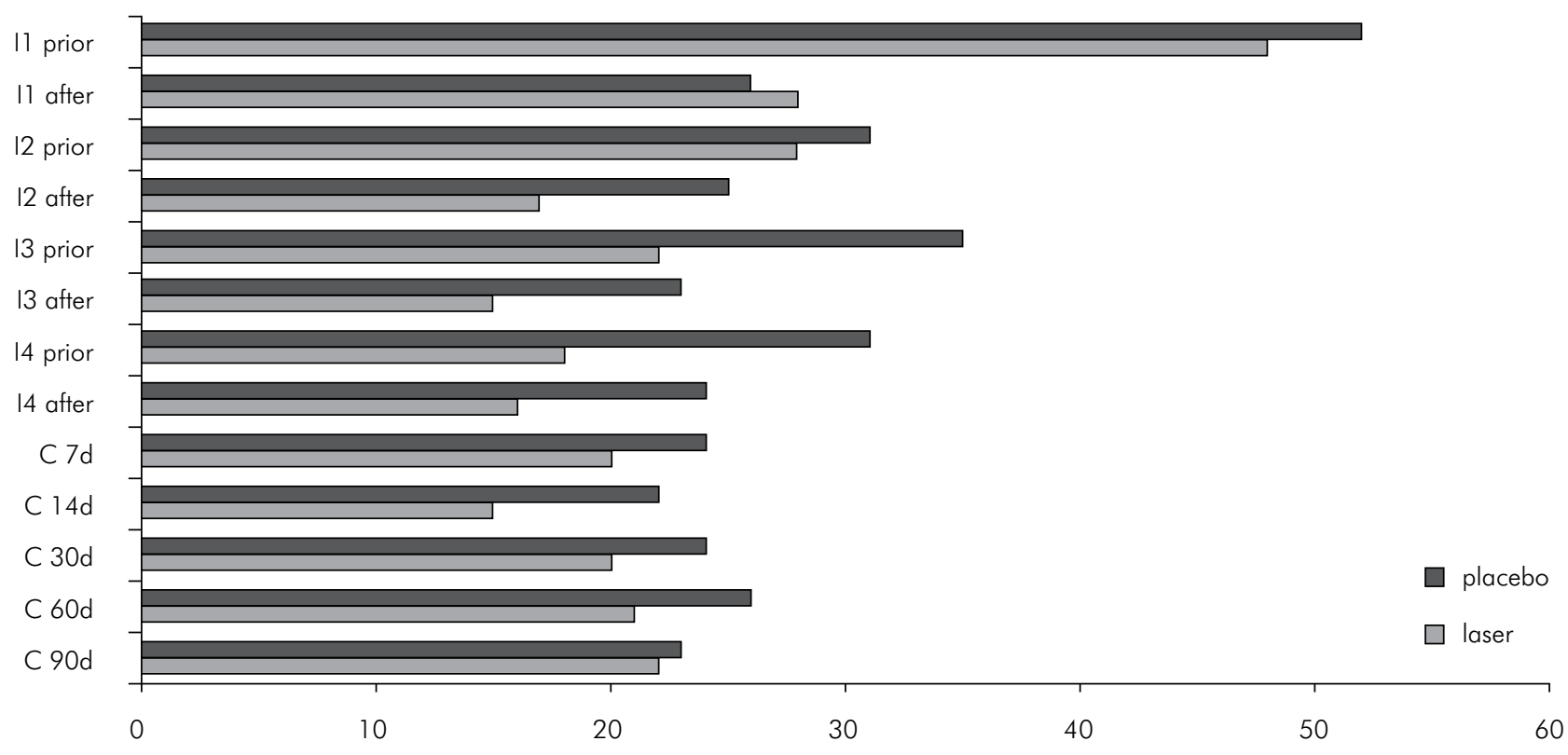

$11-4=$ irradiations sessions 1 to 4 ; prior= immediately before irradiation; after $=$ immediately after irradiation; $C=$ control checkpoints; $d=$ days after last irradiation .

Figure 2. Mean VAS values of both groups (LG, CG) in all irradiation sessions and control checkpoints. 
conduction block and modulation of neurotransmitters of A $\delta$ and C nociceptors is temporary when a laser is applied in a single session, but these effects last longer when laser irradiation is performed in several sessions.

A reduced number of sessions were chosen in order to perceive the potentiality of the technique. It is known that low intensity laser therapy applied to superficial inflammatory processes (such as mucositis caused by chemotherapy and radiotherapy) in a single session produces immediate analgesic effects ${ }^{24}$. There is a theory suggesting that neurogenic inflammation is the cause of $\mathrm{BMS}^{4}$. In fact, if there were a more complex alteration in the neurotransmission mechanisms in the genesis of BMS symptoms, a larger number of irradiation sessions could probably generate a more effective result.

Our sample was composed mostly of women (91.3\%) with a mean age of 59.7 years. These data are in agreement with most of the findings in literature ${ }^{1,2}$, although the age group represented by this sample was composed mainly of individuals in their $7^{\text {th }}$ decade of life. This fact may be attributed to the small size of the patient group. Most of the sample reported symptoms of dysgeusia and/or xerostomia associated with burning mouth (74\%) and spontaneous onset of BMS, similar to the reports found in the literature ${ }^{5,6}$. The tongue was the most affected site, followed by the lips and palate, also in agreement with data in the literature?

At present only anxiolytic, anticonvulsant and antidepressant drugs show some effectiveness in treating $\mathrm{BMS}^{25,26,27}$. Since these drugs are not completely effective against BMS, and also offer the risk of undesired side effects, the use of low intensity laser is an interesting therapeutic alternative, given its easy application, comfort, and absence of undesirable side effects. The disadvantages of the method include the need for several visits to the clinic and an appropriate environment for compliance with biosafety regulations.

According to the protocol used in this study, low intensity laser therapy showed a statistically significant beneficial effect over placebo in only two checkpoint controls (after the fourth irradiation session and at the 90-day control point), leading to the conclusion that only four irradiation sessions with the laser parameters used offer no significant difference between laser and placebo in treating BMS symptoms. Nevertheless, both treatments (laser and placebo) showed a fairly interesting benefit: $46 \%$ of no burning sensation after 90 days in the laser group and $40 \%$ in the placebo group, thus suggesting that emotional aspects greatly influence BMS manifestation.

Santos et al. ${ }^{28}$, treated 10 BMS patients with a 660 $\mathrm{nm}, 40 \mathrm{~mW}$ power, $20 \mathrm{~J} / \mathrm{cm}^{2}$ diode laser, one session per week for 10 weeks, and obtained a significant improvement in the symptoms of all patients. Yang and Huang ${ }^{29}$, obtained similar results treating 17 BMS patients with an $800 \mathrm{~nm}$ diode laser once a week for a period ranging from one to seven weeks. The weakness of these two trials is the lack of a control group. The results achieved were very similar to those obtained in the present study, despite the differences in the number of irradiation sessions and in the laser parameters utilized.

Arbabi-Kalati et al. ${ }^{30}$, evaluated 20 BMS patients divided in two groups (laser and placebo). Patients of the laser group were irradiated (iodine-galliumarsenide laser, $630 \mathrm{~nm}, 30 \mathrm{~mW}, 1 \mathrm{~J} / \mathrm{cm}^{2}$ ) twice a week for 2 weeks, in 10 areas of the oral mucosa. The placebo group received the same treatment with silent/off laser therapy. Results were collected at the end of the two-week treatment. The laser group presented a statistically better result at VAS measurement. On the other hand, Pezelj-Ribarić et al. ${ }^{31}$, treated 40 BMS patients equally divided into two groups (laser and placebo). Patients of the laser group were treated 5 days a week for 4 consecutive weeks, with a $685 \mathrm{~nm}$ galliumaluminum-arsenic diode laser $\left(30 \mathrm{~mW}, 3 \mathrm{~J} / \mathrm{cm}^{2}\right)$. The authors found no difference in symptomatology between the two groups. The treatment protocols of these two studies used wavelengths smaller than ours, as well as different laser parameters.

Arduino et al. ${ }^{32}$, treated 33 BMS patients randomly allocated to two groups: one received two irradiation laser sessions ( $980 \mathrm{~nm}$ AsGaAl diode laser, $300 \mathrm{~mW}, 10 \mathrm{~J} / \mathrm{cm}^{2}$ ) per week for 5 weeks, and the other received topical clonazepam therapy (1 $\mathrm{mg}$ tablet) orally, without swallowing for 3 minutes, three times a day for 21 days. The laser group experienced a major decrease in pain sensation, compared with the clonazepam group, up to 12 weeks after the end 
of the protocol, but this difference was statistically different only at the 8-week checkpoint. The authors concluded that LLLT is capable of reducing the symptoms of patients with BMS.

Vucicevic Boras et al. ${ }^{33}$, revised the files of 187 patients with BMS submitted to diverse kinds of therapy: salivary substitutes, chlorhexidine mouthwash, vitamin B replacement therapy and low level laser therapy. One hundred and seventeen patients (62.6\%) reported their condition as unchanged, whereas $70(37.4 \%)$ patients reported improvement compared with the baseline. None of the patients reported complete resolution of the symptoms.

\section{References}

1. Grushka M, Epstein JB, Gorsky M. Burning mouth syndrome: differential diagnosis. Dermatol Ther (Heidelb). 2002;15(3):287-91. doi:10.1046/j.1529-8019.2002.01535.x

2. Bergdahl J, Anneroth G. Burning mouth syndrome: literature review and model for research and management. J Oral Pathol Med. 1993;22(10):433-8. doi:10.1111/j.1600-0714.1993.tb00120.x

3. Grushka M, Sessle BJ. Burning mouth syndrome. Dent Clin North Am. 1991;35(1):171-84.

4. Borelli V, Marchioli A, Di Taranto R, Romano M, Chiandussi $S$, Di Lenarda R et al. Neuropeptides in saliva of subjects with burning mouth syndrome: a pilot study. Oral Dis. 2010;16(4):365-74. doi:10.1111/j.1601-0825.2009.01648.x

5. Ship JA, Grushka M, Lipton JA, Mott AE, Sessle

BJ, Dionne RA. Burning mouth syndrome: an update. J Am Dent Assoc. 1995;126(7):842-53. doi:10.14219/jada.archive.1995.0305

6. Nagler RM, Hershkovich O. Sialochemical and gustatory analysis in patients with oral sensory complaints. J Pain. 2004;5(1):56-63. doi:10.1016/j.jpain.2003.09.002

7. Grushka M. Clinical features of burning mouth syndrome. Oral Surg Oral Med Oral Pathol. 1987;63(1):30-6. doi:10.1016/0030-4220(87)90336-7

8. Scala A, Checchi L, Montevecchi M, Marini I, Giamberardino MA. Update on burning mouth syndrome: overview and patient management. Crit Rev Oral Biol Med. 2003;14(4):275-91. doi:10.1177/154411130301400405

9. Sardella A, Lodi G, Demarosi F, Bez C, Cassano S, Carrassi A. Burning mouth syndrome: a retrospective study investigating spontaneous remission and response to treatments. Oral Dis. 2006;12(2):152-6. doi:10.1111/j.1601-0825.2005.01174.x

10. Suarez P, Clark GT. Burning mouth syndrome: an update on diagnosis and treatment methods. J Calif Dent Assoc. 2006;34(8):611-22.

\section{Conclusion}

The laser protocol used to treat this group of BMS patients produced benefits similar to those of the placebo treatment applied. Considering the beneficial effect obtained, versus the extremely different results reported in the literature, further research in BMS laser therapy is still valid to improve the knowledge in this field, and to help clarify the mechanisms involved in BMS etiopathogenesis.

\section{Acknowledgements}

This investigation was supported by the State of Sao Paulo Research Foundation (FAPESP; grant 08/56339-9).

11. Forssell H, Jääskeläinen S, Tenovuo O, Hinkka S. Sensory dysfunction in burning mouth syndrome. Pain. 2002;99(1-2):41-7. doi:10.1016/S0304-3959(02)00052-0

12. Jääskeläinen SK. Pathophysiology of primary burning mouth syndrome. Clin Neurophysiol. 2012;123(1):71-7. doi:10.1016/j.clinph.2011.07.054

13. Lauria G, Majorana A, Borgna M, Lombardi R, Penza $\mathrm{P}$, Padovani A et al. Trigeminal small-fiber sensory neuropathy causes burning mouth syndrome. Pain. 2005;115(3):332-7. doi:10.1016/j.pain.2005.03.028

14. Eliav E, Kamran B, Schaham R, Czerninski R, Gracely RH, Benoliel R. Evidence of chorda tympani dysfunction in patients with burning mouth syndrome. J Am Dent Assoc. 2007;138(5):628-33. doi:10.14219/jada.archive.2007.0234

15. Zidverc-Trajkovic J, Stanimirovic D, Obrenovic R, Tajti J, Vécsei L, Gardi J et al. Calcitonin gene-related peptide levels in saliva of patients with burning mouth syndrome. J Oral Pathol Med. 2009;38(1):29-33. doi:10.1111/j.1600-0714.2008.00721.x

16. Rumsfield JA, West DP. Topical capsaicin in dermatologic and peripheral pain disorders. DICP. 1991;25(4):381-7.

17. Cavalcanti DR, Silveira FRX. Alpha lipoic acid in burning mouth syndrome-a randomized double-blind placebo-controlled trial. J Oral Pathol Med. 2009;38(3):254-61. doi:10.1111/j.1600-0714.2008.00735.x

18. Kato IT, Pellegrini VD, Prates RA, Ribeiro MS, Wetter NU, Sugaya NN. Low-level laser therapy in burning mouth syndrome patients: a pilot study. Photomedicine Laser Surg. 2010;28(6):835-9. doi:10.1089/pho.2009.2630

19. Olsen JE, Schimmerling W, Tobias CA. Laser action spectrum of reduced excitability in nerve cells. Brain Res. 1981;204(2):436-40. doi:10.1016/0006-8993(81)90604-1 
20. Miloro M, Halkias LE, Mallery S, Travers S, Rashid RG. Low-level laser effect on neural regeneration in Gore-Tex tubes. Oral Surg Oral Med Oral Pathol Oral Radiol Endod. 2002;93(1):27-34. doi:10.1067/moe.2002.119518

21. Rochkind S. Photoengineering of neural tissue repair processes in peripheral nerves and the spinal cord: research development with clinical applications. Photomed Laser Surg. 2006;24(2):151-7. doi:10.1089/pho.2006.24.151

22. Rochkind S, Drory V, Alon M, Nissan M, Ouaknine GE. Laser phototherapy $(780 \mathrm{~nm})$, a new modality in treatment of long-term incomplete peripheral nerve injury: a randomized double-blind placebo-controlled study. Photomed Laser Surg. 2007;25(5):436-42. doi:10.1089/pho.2007.2093

23. Chow RT, Heller GZ, Barnsley L. The effect of $300 \mathrm{~mW}$, $830 \mathrm{~nm}$ laser on chronic neck pain: a double-blind, randomized, placebo-controlled study. Pain. 2006;124(12):201-10. doi:10.1016/j.pain.2006.05.018

24. Huang YY, Chen AC, Carroll JD, Hamblin MR. Biphasic dose response in low level light therapy. Dose Response. 2009;7(4):358-83. doi:10.2203/dose-response.09-027.Hamblin

25. Gremeau-Richard C, Woda A, Navez ML, Attal N, Bouhassira D, Gagnieu MC et al. Topical clonazepam in stomatodynia: a randomised placebo-controlled study. Pain. 2004;108(1-2):51-7. doi:10.1016/j.pain.2003.12.002

26. Barker KE, Batstone MD, Savage NW. Comparison of treatment modalities in burning mouth syndrome. Aust Dent J. 2009;54(4):300-5. doi:10.1111/j.1834-7819.2009.01154.x
27. Yamazaki Y, Hata H, Kitamori S, Onodera M, Kitagawa Y. An open-label, noncomparative, dose escalation pilot study of the effect of paroxetine in treatment of burning mouth syndrome. Oral Surg Oral Med Oral Pathol Oral Radiol Endod. 2009;107:e6-11. doi:10.1016/j.tripleo.2008.08.024

28. Santos LFC, Carvalho AAT, Leão JC, Perez DEC, Castro JFL. Effect of low-level laser therapy in the treatment of burning mouth syndrome: a case series. Photomed Laser Surg. 2011;29(12):793-6. doi:10.1089/pho.2011.3016

29. Yang HW, Huang YF. Treatment of burning mouth syndrome with a low-level energy diode laser. Photomed Laser Surg. 2011;29(2):123-5. doi:10.1089/pho.2010.2787

30. Arbabi-Kalati F, Bakhshani N, Rasti M. Evaluation of the efficacy of low-level laser in improving the symptoms of burning mouth syndrome. J Clin Exp Dent. 2015;7(4):e524-7. doi:10.4317/jced.52298

31. Pezelj-Ribarić S, Kqiku L, Brumini G, Urek MM, Antonić $\mathrm{R}, \mathrm{Kuiš} \mathrm{D} \mathrm{et} \mathrm{al.} \mathrm{Proinflammatory} \mathrm{cytokine} \mathrm{levels} \mathrm{in} \mathrm{saliva}$ in patients with burning mouth syndrome before and after treatment with low-level laser therapy. Lasers Med Sci. 2013;28(1):297-301. doi:10.1007/s10103-012-1149-5

32. Arduino PG, Cafaro A, Garrone M, Gambino A, Cabras $\mathrm{M}$, Romagnoli E et al. A randomized pilot study to assess the safety and the value of low-level laser therapy versus clonazepam in patients with burning mouth syndrome. Lasers Med Sci. 2016;31(4):811-6. doi:10.1007/s10103-016-1897-8

33. Vucicevic Boras V, Peakic M, Music L, Krpan K, Illes D, Gabric D. Burning mouth syndrome: retrospective analysis of 328 patients. Res J Pharm Biol Chem Sci. 2015;6(5):141-6. 